\title{
Un análisis del proceso de aprendizaje social en animales no humanos: situaciones apetitivas versus aversivas*
}

\author{
An Analysis of Social Learning Process in No Human \\ Animals: Appetitive Versus Aversive Settings
}

Recibido: enero 13 de 2011 | Revisado: julio 14 de 2011 | Aceptado: noviembre 13 de 2011

\author{
ROSALVA CABrera** \\ Universidad Nacional Autónoma de México \\ CRISTIANO V. DOS SANTOS *** \\ Universidad de Guadalajara, Jalisco, México
}

\section{RESUMEN}

El objetivo es identificar si los elementos que definen diferentes procesos de aprendizaje social, tanto imitativo como no imitativo, discutidos y evaluados en situaciones apetitivas pueden ser identificados en situaciones aversivas. Esta identificación permitirá valorar las bondades y/o limitaciones de los términos definidos, así como su alcance conceptual y heurístico. Se iniciará con un análisis general del concepto de aprendizaje, seguido de la distinción entre aprendizaje individual y social; posteriormente, se hará una delimitación de los componentes involucrados en los diferentes procesos de aprendizaje social en situaciones apetitivas, señalando las dificultades tanto conceptuales como metodológicas inherentes a esta área de investigación; se continuará con la misma delimitación de procesos en situaciones aversivas y se terminará con una discusión en la que, además de comparar las preparaciones experimentales en situaciones apetitivas y aversivas, se valorará si pueden ser descritas con los mismos conceptos.

Palabras clave autores:

Apetitivo, aprendizaje social, aversivo, conceptual, experimental.

Palabras clave descriptores:

Análisis Experimental de la Conducta, aprendizaje, revisión.

Para citar este artículo: Cabrera, R. \& dos Santos, C. V. (2012). Un análisis del proceso de aprendizaje social en animales no humanos: situaciones apetitivas versus aversivas. Universitas Psychologica, 11(3), 989-1000

Rosalva Cabrera agradece a la Facultad de Estudios Superiores Iztacala (UNAM) el año sabático otorgado y al CEIC (Universidad de Guadalajara) la Estancia Sabática que permitió la realización de este manuscrito.

** Facultad de Estudios Superiores Iztacala (UNAM). Ave. de los Barrios № 1, Los Reyes Iztacala. Tlalnepantla, Estado de México. México 56090. Tel. 56231333.Email: rosalva@unam.mx

**** CEIC (Universidad de Guadalajara). Francisco de Quevedo № 180, Colonia Arcos Vallarta. Guadalajara, Jalisco. México 44130. Tel. 38180730. Email: behaca@yahoo.com

\section{A B S T R A C T}

This paper attempts to identify whether the components involved in different processes of social learning, both imitative and non imitative, evaluated in appetitive contexts can be identified in an aversive context. This identification will allow us to appraise the goodness and/or limitations of the concepts and their heuristic value. The paper begins with the definitions of learning, individual learning and social learning, followed by the definition of the different processes of social learning in appetitive contexts, with emphasis in conceptual and experimental difficulties. Then, the definition of the different processes of social learning in aversive context is proposed and the paper concludes with a discussion about differences and similarities in both contexts.

Key words authors:

Appetitive, Aversive, Conceptual, Experimental, Social Learning.

Key words plus:

Experimental Analysis of Behavior, Learning, Review. 


\section{Introducción}

El aprendizaje es un proceso que ha sido ampliamente estudiado en el análisis experimental de la conducta, de tal manera que existe una gran cantidad de trabajos empíricos en los que se ha identificado el efecto preciso de diferentes variables sobre una amplia gama de cambios conductuales manifiestos en los organismos sujetos a experimentación (Catania, 1989; Honig, 1966; Honig \& Staddon, 1977), lo cual ha permitido que las relaciones funcionales observadas entre las variables involucradas sean expresadas formalmente, como en el modelo de Rescorla y Wagner (1972) o en la ley de igualación de Herrnstein (1970).

Gran parte de este trabajo se ha realizado exponiendo a los organismos de manera individual a las diferentes condiciones experimentales; sin embargo, existen trabajos en los cuales al menos dos organismos son expuestos de manera simultánea a la situación experimental y cuyo interés central radica en evaluar el papel que juega la presencia de un coespecífico en la adquisición de una respuesta novedosa, proceso denominado como aprendizaje social.

Desde la perspectiva de la Psicología experimental, Thorndike (1898) fue el primero en realizar investigaciones de laboratorio para evaluar si diferentes especies animales son capaces de aprender cuando observan a otro sujeto ejecutar una respuesta.

Posteriormente, las investigaciones en laboratorio sobre aprendizaje social fueron retomadas, permitiendo la identificación de diferentes procesos conductuales, cada uno de los cuales implica una relación funcional diferente entre la actividad mostrada por un sujeto demostrador y la subsecuente actividad del sujeto observador. A pesar de que hay un número importante de trabajos experimentales y de discusiones conceptuales, aún no existe completa claridad acerca de los elementos involucrados en esa relación funcional y menos aún del peso relativo que tiene cada uno de ellos. De esta manera, se tienen problemas tanto para definir los diferentes procesos de aprendizaje social de manera excluyente como para delimitar experimentalmente los elementos involucrados (Avital \& Jablonka, 1994; Heyes, 1994; Nicol \& Pope, 1992; Whiten \& Ham, 1992).

\section{Aprendizaje}

Algunas teorías contemporáneas sobre el aprendizaje lo conciben como un cambio en la conducta de un organismo que es producido por una experiencia específica ocurrida en un tiempo particular (t1) y que es demostrado en un tiempo posterior ( $\mathrm{t} 2$ ). Así, el aprendizaje ha sido categorizado con relación al tipo de experiencia que produce el cambio en el organismo y al tipo de cambio conductual que es observado (Dickinson, 1980; Heyes, 1994; Mackintosh, 1983; Rescorla, 1988).

Heyes (1994) propone que el aprendizaje social, al igual que el aprendizaje individual o asocial, puede ser categorizado con base en el tipo de experiencia a que es expuesto el organismo en $\mathrm{t} 1$ y al cambio conductual que se produce en $\mathrm{t} 2$ como resultado de esta experiencia. Su categorización empieza con la distinción entre dos grandes instancias de aprendizaje social: no imitativo, cuando el cambio conductual en el sujeto observador es resultado de que la conducta del demostrador atrajo su atención a un lugar u objeto particular, e imitativo, cuando el cambio conductual en el observador consiste en ejecutar una respuesta de igualación que es resultado de la observación de una relación de contingencia entre la respuesta de un demostrador y el subsecuente reforzamiento.

\section{El aprendizaje social en situaciones apetitivas}

Las categorías de aprendizaje social no imitativo que han sido definidas y evaluadas en situaciones apetitivas son:

1) Contagio: se presenta cuando en el t $1 \mathrm{el} \mathrm{ob}$ servador es expuesto a un coespecífico y en el t 2 el observador emite una clase de respuesta idéntica a la que realizó su coespecífico previamente, y esta igualación de respuestas es limitada a aquellas respuestas que son típicas de las especies, tales como el inicio y propagación de "canto" en las aves o el cortejo sincronizado. 
2) Facilitación social: es el proceso en el que las respuestas que no son típicas de la especie son influidas por la sola presencia de un coespecífico, de tal manera que un observador que es expuesto a un coespecífico emitirá con mayor probabilidad una respuesta que sujetos que no tienen esta experiencia. Un ejemplo clásico de ese proceso es el estudio de Zajonc (1965), en el cual cucarachas corrieron con mayor velocidad en presencia de coespecíficos. Posteriormente, Zentall y Hogan (1976) realizaron un experimento en el que palomas acompañadas de un coespecífico emitieron con mayor probabilidad la respuesta de picar una tecla que sujetos expuestos en solitario a la situación experimental.

3) Realce de estímulos: se habla de este proceso (Zentall \& Levine, 1972) si la exposición al demostrador en $\mathrm{t} 1$ aumenta la probabilidad de que el observador dirija su atención a un estímulo (por ejemplo, un manipulandum) en $\mathrm{t} 2$.

4) Realce local: se habla de la presencia de un proceso de realce local si el observador es expuesto a un demostrador entrenado y ello produce un incremento en la probabilidad de que el observador atienda a una parte específica de la situación asociada a reforzamiento. Por ejemplo, si un observador ve a un modelo presionando una palanca, es posible que el observador aprenda a ejecutar la respuesta, porque se dirige a la zona donde estaba el modelo y esa cercanía propicia que interactúe con la palanca, mas no porque haya aprendido a presionar la palanca a través de la conducta del demostrador.

5) Condicionamiento observacional: si la observación de un demostrador expone al observador a una relación entre estímulos en tl y esta exposición evoca una respuesta de igualación en el observador en $\mathrm{t} 2$ se le denomina condicionamiento observacional. Si un observador es expuesto a un demostrador que al picar una tecla iluminada recibe alimento y posteriormente este observador también pica la tecla iluminada, la respuesta del observador puede ser función de la asociación entre la luz de la tecla y la consecuente presentación de alimento, más que de la respuesta del demostrador a la tecla.

6) Copia: en este proceso solo es necesario que el demostrador emita una respuesta para que el observador la iguale, tal es el caso de vocalizaciones arbitrarias en algunas aves, como en el caso de los pericos (Galef, 1988; Heyes, 1994; Heyes, 1996a, 1996b; Zentall, 1996).

Por su parte, las categorías de aprendizaje social imitativo son:

1) Emulación de la meta: cuando el observador reproduce los resultados de la conducta del demostrador más que la conducta misma, se habla de un proceso de emulación de la meta. Klein y Zentall (2003) diseñaron un procedimiento de control al que denominan affordance para descartar que, al emitir una respuesta novedosa, los sujetos lo estén haciendo porque ello les permite acceder a la consecuencia y no porque hayan visto a un demostrador ejecutar esta respuesta y producir la consecuencia. En su experimento, los observadores del grupo experimental -imitación- vieron a un congénere desplazar una compuerta a la derecha o a la izquierda para dejar disponible el alimento contenido en un dispensador, mientras que los sujetos del grupo control-affordance- ven que la compuerta se desplaza automáticamente a un lado o al otro, quedando disponible el alimento; si en la prueba los sujetos del grupo affordance igualaran la dirección izquierda/derecha del desplazamiento de la compuerta, lo estarán haciendo solo porque les permite acceder a la meta. En este experimento, los sujetos del grupo affordance emitieron una proporción mayor de respuestas de igualación que los sujetos de los grupos que no vieron desplazamiento alguno de la compuerta, lo cual sugiere que el mero desplazamiento, sin la observación de la respuesta de parte de un congénere, puede ser suficiente para que un sujeto iguale la respuesta "observada".

2) Aprendizaje por observación o imitación: cuando la observación de un demostrador expone al observador a una relación respuesta-reforzador en t1 y esta observación produce un cambio en la conducta del observador en $\mathrm{t} 2$, se le denomina aprendizaje por observación o imitación (Galef, 1988; Heyes, 1994, 1996a, 1996b; Zentall, 1996). En el ejemplo anterior de Klein y Zentall (2003), si los observadores del grupo experimental emitieran una respuesta de igualación con mayor probabilidad que los sujetos de los grupos control, se demostraría que su respuesta es función de su exposición a una 
relación respuesta-consecuencia. Los autores concluyen que hubo aprendizaje por observación porque su grupo experimental, que vio a un congénere desplazar la compuerta en una dirección, igualó la dirección de la respuesta en mayor proporción que los grupos control. Sin embargo, aun cuando Klein y Zentall (2003) hayan argumentado que sus datos proporcionan evidencia de aprendizaje imitativo, el efecto por ellos reportado parece débil, ya que la diferencia entre el grupo affordance y el grupo experimental no fue estadísticamente significativa.

\section{El aprendizaje social en situaciones aversivas}

La expresión control aversivo se refiere usualmente a los procedimientos de reforzamiento negativo y a los dos tipos de castigo, tanto el positivo como el negativo. En el caso del reforzamiento negativo, tradicionalmente dividido en evitación y escape, el organismo emite una respuesta que elimina, cancela o pospone un estímulo y, como consecuencia, la frecuencia con que esa respuesta ocurre aumenta. En el caso del castigo, la frecuencia de una respuesta disminuye como consecuencia de que esta produce un estímulo, generalmente llamado aversivo (castigo positivo), o de que conlleva a la remoción de algún estímulo apetitivo (castigo negativo).

Las respuestas analizadas en los estudios sobre control aversivo con animales no humanos son generalmente las mismas estudiadas con reforzamiento positivo, a saber, presionar palancas o pedales, picar discos iluminados, jalar cadenas, correr (en laberintos, shuttleboxes o ruedas de actividad), brincar barreras, entre otras. El principal estímulo usado en esos procedimientos ha sido la descarga eléctrica, administrada por el piso de metal o por electrodos implantados en la piel (Iversen \& Lattal, 1991).

A continuación, se tratará de identificar los procesos de aprendizaje social descritos en la sección anterior en situaciones de control aversivo, iniciando con las categorías no imitativas:

1) Contagio. En situaciones aversivas, al igual que en apetitivas, el demostrador produce una respuesta y esta se propaga en el observador. Papworth, Böse, Barker, Schel y Zuberbühler (2008) identificaron que llamados de alarma en monos azules se propagan entre los miembros de un grupo ante la identificación de un predador y que esos llamados son diferentes en función del tipo de predador. De este modo, en t1, los monos escuchan el llamado de alarma emitido por un coespecífico y, en $\mathrm{t} 2$, lo reproducen.

2) También se puede observar facilitación social en situaciones aversivas; por ejemplo, el estudio de Sanavio y Savardi (1979) donde se pretendía identificar si las ratas podrían aprender una respuesta de evitación después de observar a otra rata haciendo una respuesta para posponer la presentación de una descarga eléctrica. Un grupo fue expuesto a un demostrador que evitaba las descargas brincando una barrera en una shuttlebox mientras se presentaba un tono; el segundo grupo fue expuesto a un modelo no entrenado en la respuesta de brincar para evitar la descarga durante la presentación del tono; el tercer grupo no fue expuesto a demostrador, solo a la presentación del tono. Después de esta fase, los observadores fueron expuestos a una prueba que consistía en colocarlos en la shuttlebox presentándoles un tono. Si el sujeto emitía la respuesta de brincar, el choque se posponía; si el sujeto no emitía la respuesta, el choque se presentaba. Si el sujeto respondía estando presente el choque podía escapar de él. El análisis de los resultados señala que los sujetos expuestos a un demostrador que saltaba para evitar el choque ejecutaron con mayor frecuencia y con latencias más cortas las respuestas de saltar que los sujetos expuestos a un modelo no entrenado. Sin embargo, los sujetos que no fueron expuestos a demostradores tuvieron una mejor ejecución que los sujetos expuestos al demostrador no entrenado. Sanavio y Savardi (1979) atribuyen los resultados al proceso de facilitación social, ya que la simple presencia de otro sujeto puede disminuir el miedo generado por los choques y por lo tanto, retardar la ejecución de la respuesta.

3) Para hablar de realce de estímulos o local en situaciones aversivas, sería necesario que un demostrador señalara un estímulo relevante y que ello facilitara la interacción posterior del observador con ese estímulo, permitiéndole evitar o escapar de una situación aversiva, lo cual, de principio, parece ser incongruente, dado que es difícil suponer que 
el resaltamiento de un estímulo relevante produzca que el sujeto no interactúe con el mismo.

4) En el caso de condicionamiento observacional en situaciones aversivas, la exposición del observador en $\mathrm{t} 1 \mathrm{a}$ un demostrador que es expuesto a una relación entre un estímulo neutral y un estímulo aversivo, debe facilitar las respuestas de evitación o escape cuando el observador recibe directamente el estímulo aversivo en t2. Riess (1972) realizó un experimento en el cual ratas observadoras vieron una luz y escucharon los chillidos de una rata demostradora que recibía descargas eléctricas inevitables; posteriormente, las observadoras fueron expuestas a una contingencia de evitación en la cual podían saltar a un compartimiento seguro para evitar la descarga. Las ratas observadoras mostraron una aceleración en su tasa de respuestas en presencia de la luz, lo cual demuestra que fueron sensibles a la relación entre la luz y los efectos de la descarga en la demostradora.

5) Dado que la copia se caracteriza únicamente por la reproducción de una respuesta sin relación con consecuencia alguna, no tiene sentido distinguir entre la ocurrencia de una respuesta de copia en situaciones apetitivas versus aversivas.

Las categorías imitativas son:

1) En emulación de meta, el observador atiende a la consecuencia que produce la conducta del demostrador más que a la conducta en sí misma y, por ello, emitirá aquellas respuestas que le permitan obtener la misma consecuencia, las cuales pueden o no igualar la respuesta del demostrador. Cuando el contexto experimental es aversivo, la consecuencia puede consistir en estar en una situación de seguridad; la definición de emulación de meta implica que el observador que ha visto a un demostrador en una situación segura tratará de estar en la misma condición cuando es expuesto a estímulos aversivos. Así, la evaluación experimental de emular una meta aversiva puede requerir que el observador sea pre-expuesto a la presentación del estímulo aversivo en un contexto $\mathrm{A}$ y que posteriormente observe que el demostrador no recibe el estímulo aversivo en un contexto B. Si, en esta situación, vemos que el observador emite respuestas para llegar al contexto B sin haber visto al demostrador emitiendo cualquier respuesta, entonces hablaríamos de emulación de meta.

2) El aprendizaje por observación aversivo requiere que el observador vea a un demostrador ejecutar una respuesta que le permita suprimir un estímulo aversivo y que, posteriormente, el observador realice la misma respuesta y produzca la misma consecuencia.

Como resulta fácil advertir, la sola transposición de los elementos considerados en situaciones apetitivas a situaciones aversivas es en sí misma problemática; ello obedece en buena medida al hecho de que en muchas ocasiones la consecuencia en situaciones aversivas consiste en la ausencia de un estímulo, la cual no puede ser vista directamente por el observador.

Por ejemplo, en los experimentos de Cook y Mineka (1990) sobre condicionamiento observacional de la respuesta de miedo, un mono ingenuo es expuesto, en un primer momento (t1), al video de otro mono emitiendo respuestas típicas de miedo (e.g., agarrarse a la jaula, golpearla, etc.) ante algún objeto (e.g., una serpiente, un juguete o una flor). En esos casos, el observador, al ser expuesto a algunos de los objetos en una fase posterior ( $\mathrm{t} 2$ ), presenta las respuestas típicas de miedo similares a las del demostrador. Esos experimentos son analizados como ejemplo de condicionamiento observacional.

Sin embargo, en el caso del condicionamiento observacional apetitivo, el observador percibe un estímulo neutral (por ejemplo, el sonido de un microswitch), ve la consecuente presentación de alimento-sin observar la respuesta que lo producey las subsecuentes respuestas consumatorias, de tal manera que hay una asociación entre el estímulo neutral y un estímulo reforzador, lo cual amerita la clasificación de condicionamiento observacional. En el caso de los experimentos de Cook y Mineka (1990), el observador ve una o más respuestas típicas de miedo y un estímulo neutral asociado a las respuestas (por ejemplo, la serpiente), pero no observa el estímulo incondicionado (el veneno o la estrangulación).

Lo mismo ocurre en experimentos de aversión a olores transmitida socialmente (Bond, 1984; Coombs, Revusky \& Lett, 1980; Lavin, Freise \& 
Coombs, 1980). En estos, un sujeto es retirado de un grupo y se le permite comer algo que exhale un olor característico (e.g., menta). Luego, se le aplica una inyección de cloruro de litio, sin que los otros miembros del grupo lo vean. Cuando regresa al grupo, presenta una serie de respuestas asociadas a malestar estomacal causado por el cloruro de litio (t1). Como resultado de esa experiencia, los miembros del grupo que observaron las reacciones de malestar asociadas a un olor característico evitaron comidas que exhalaron ese olor en un segundo momento ( $t 2)$.

Aquí, nuevamente se nota que los observadores ven algunas respuestas asociadas a algún estímulo neutral, pero no el estímulo incondicionado y, por ende, no observan una relación estímulo-estímulo que sería característica del condicionamiento observacional. Los elementos presentes en esas situaciones serían equivalentes, en procedimientos apetitivos, a que el observador viera un estímulo neutral, no observara ni la respuesta que produce la comida ni la comida misma, pero observara la respuesta de consumir el alimento y sus consecuencias postingestivas.

En el condicionamiento operante, el análisis se complica aún más, dada la conocida dificultad en identificar la consecuencia que mantiene las conductas de evitación y escape, o en los casos en que una conducta es castigada. Algunos autores como Domjam (2009) proponen que evitación y castigo son procesos que comparten el que los sujetos aprenden a minimizar su exposición al estímulo aversivo; de tal manera que cuando los organismo emiten una respuesta y no tienen contacto con el estímulo aversivo - evitación- puede hablarse de una evitación activa, mientras que cuando los sujetos no emiten una respuesta y no tienen contacto con un estímulo aversivo -castigo- puede hablarse de una evitación pasiva. Mowrer y Lamoreaux (1942) plantearon el interrogante de icómo la ausencia de algo puede reforzar la conducta instrumental?; posteriormente, Mowrer (1947) propuso que en estos casos la conducta es reforzada por una reducción del temor, vía un proceso de condicionamiento clásico y un proceso de condicionamiento operante.

En un ejemplo de castigo y escape transmitidos socialmente, Bunch y Zentall (1980) expusieron un grupo de ratas a un demostrador que tenía contacto con una vela encendida y se quemaba; los observadores podían escuchar los sonidos característicos de dolor y percibir el olor de quemadura. Un segundo grupo fue expuesto a un demostrador y a la vela encendida, pero el demostrador no tenía contacto con la vela y, por tanto, no se quemaba ni emitía sonidos. Un tercer grupo fue expuesto a un demostrador que tenía contacto con la vela, como el primer grupo, pero una pared oscura impedía la visibilidad del demostrador, por lo que el observador solo escuchaba sus sonidos y percibía el olor. Los sujetos del primer grupo tuvieron menor número de contactos con la vela que los demás.

En este procedimiento, los elementos con los cuales el observador del primer grupo entra en contacto durante la fase de observación (t1) son una vela encendida, un demostrador que emite una respuesta (acercarse a la vela y tocarla), los sonidos característicos de dolor emitidos por el demostrador y la consecuente respuesta de alejamiento de la vela, los cuales indican que la respuesta de acercarse a la vela y tocarla fue castigada y la conducta de alejarse, reforzada negativamente. En ese último ejemplo, se podría argumentar que el calor emanado por la vela y no la flama de la vela como tal es la variable motivacional que aumenta la probabilidad de la respuesta que tiene como consecuencia un período de seguridad, el cual es el evento reforzante. En este caso, el observador sigue sin ver el estímulo motivacional y ve solamente las respuestas evocadas por ese estímulo. Los autores argumentan que el primer grupo fue expuesto a una relación visual respuesta-reforzador, y que por ello puede considerarse un proceso de imitación. Sin embargo, Heyes (1994) clasificó a este grupo como condicionamiento observacional, dada la similitud con el procedimiento de Cook y Mineka (1990), en el cual hay una supuesta asociación entre un estímulo (serpiente) y otro (respuestas de miedo por parte del demostrador), de tal manera que, en el procedimiento de Bunch y Zentall, la vela sería un estímulo asociado a los estímulos que proveen las respuestas de dolor del demostrador. Por otro 
lado, ambas clasificaciones pueden ser problemáticas, si se considera que el observador no ve la consecuencia o el estímulo incondicionado en ninguno de los dos casos.

$\mathrm{Al}$ segundo grupo, lo consideran como un control que evalúa un proceso de facilitación social. Sin embargo, el coespecífico no estuvo presente durante la adquisición de la evitación pasiva por parte de los observadores, así que es difícil que esa situación pueda ser clasificada como facilitación social. Finalmente, el tercer grupo, expuesto solamente a los chillidos y al olor, solo tiene contacto con un resultado de la variable motivacional sin tener oportunidad de ver ni la respuesta ni la consecuencia.

En un experimento no publicado, del laboratorio de uno de los autores, un demostrador fue expuesto a una contingencia de escape en una shuttlebox: al recibir el choque por el piso, el demostrador podía saltar hacia una ventana, empujar la puerta de la ventana y llegar a un compartimiento seguro. Los observadores del grupo experimental fueron expuestos a la siguiente secuencia de eventos: el chillido del demostrador al recibir el choque, la respuesta de salto hacia la ventana, la cual producía un feedback (movimiento de la puerta de la ventana) y finalmente el cese del chillido en el compartimiento seguro y el aumento de la actividad general del demostrador. Posteriormente, los observadores fueron expuestos a la misma contingencia de escape que su demostrador. Grupos de control vieron solo una parte de la secuencia de eventos a que fue expuesto el demostrador: así, un segundo grupo, en ausencia de un demostrador, fue expuesto a un chillido grabado seguido del movimiento de la ventana y al posterior cese del chillido; un tercer grupo fue expuesto solo al movimiento de ventana, también en ausencia de demostrador y un cuarto grupo no tuvo experiencia de observación, solo fue expuesto a la prueba. El primer y el segundo grupo presentaron más respuestas de escape que los otros dos.

En el caso del grupo experimental, se considera que es una situación de aprendizaje por observación. El choque recibido por el demostrador funge como la variable motivacional que produce la respuesta incondicionada del chillido y que aumenta la probabilidad de la respuesta de saltar; la respuesta de salto produce de manera inmediata la retroalimentación del movimiento de la ventana y el consecuente cese de la recepción del choque y de la emisión del chillido. Por lo tanto, el observador, aun cuando no vea el choque, ve a un coespecífico emitiendo una respuesta específica de la especie ante un estímulo doloroso, la respuesta instrumental de saltar, la retroalimentación inmediata de esta respuesta, y la consecuente ausencia del chillido, que de manera indirecta señala la ausencia del estímulo doloroso.

Esa situación sería análoga al aprendizaje por observación apetitivo, en el cual la privación de comida funge como variable motivacional que aumenta la actividad general del organismo. Hay una respuesta instrumental (e.g., presión de palanca o pedal), que normalmente produce una retroalimentación (cierre del interruptor) y la consecuente presencia de alimento y su respectivo consumo.

Así, el primer grupo control escuchó los chillidos de una rata, vio el movimiento de la ventana, seguido del cese del chillido; se puede considerar que este grupo pudo estar expuesto a una situación de emulación de meta, en la que estuvieron presentes una respuesta específica de la especie ante un estímulo doloroso (correspondiente a la variable motivacional del grupo experimental), el movimiento de la puerta de la ventana (correspondiente a la retroalimentación a la respuesta de salto) y el consecuente cese del chillido. Sin embargo, la respuesta blanco (saltar) no fue observada. En situaciones apetitivas de emulación de meta, los observadores ven el funcionamiento automático del operandum y la consecuente presencia de la comida y su consumo. En este experimento, el hecho de que los observadores no hayan visto la ejecución de la respuesta blanco, sino el movimiento automático de la puerta de la ventana y el cese de la respuesta asociada al estímulo doloroso, nos permite clasificar a esta situación como emulación de meta en un contexto aversivo.

El segundo grupo control, expuesto solamente al movimiento de la puerta de la ventana, pudo estar expuesto a una situación de realce de estímulos, ya que los observadores únicamente vieron el movimiento automático de la ventana. 


\section{Discusión}

A pesar de existir cierto consenso en las definiciones de diferentes procesos de aprendizaje social, la reseña previamente realizada muestra que existen incongruencias de diferente orden; algunas son inherentes a la definición misma de los procesos, otras a la confusión entre diferentes procesos y otras más son confusiones de carácter empírico.

\section{Los problemas de definición}

Relacionadas con la definición de los procesos están contagio y facilitación social, clasificados como procesos de aprendizaje social, aunque en sentido estricto no lo son, porque las respuestas analizadas muchas veces ya hacen parte del repertorio del organismo, de tal manera que no se está aprendiendo una respuesta novedosa en la situación ni una nueva direccionalidad o función de la respuesta. Asimismo, resulta difícil distinguir esos dos procesos en términos de naturaleza innata o aprendida de la respuesta, pues en algunas ocasiones, como en el caso del estudio de Zajonc (1965) que es muy citado como un ejemplo típico de facilitación social, se evaluó en cucarachas la respuesta de correr, la cual definitivamente no es aprendida en la situación experimental y, por tanto, no es novedosa.

La confusión entre definiciones también está entre realce de estímulos (o local) y condicionamiento observacional. Por ejemplo, Zentall (1996) al definir realce local, menciona que el lugar señalado por el demostrador cobra relevancia porque es asociado con reforzamiento. Por otro lado, al definir realce de estímulos, proceso que considera igual a realce local, no menciona la asociación con el reforzador como un elemento sustantivo para que el demostrador resalte el local, lo cual no es coherente con su propia definición. Además, si se considera la asociación del estímulo resaltado con el reforzador como elemento importante del proceso, tanto realce de estímulos como local se confunden con condicionamiento observacional, lo cual nos lleva a una confusión entre procesos (Heyes, 1994).

\section{Los problemas de orden empírico}

Otras dificultades al separar los diferentes procesos son de orden empírico. Por ejemplo, realce local y de estímulos son difíciles de separar, pues resulta problemático identificar qué elementos son señalados por la conducta del demostrador, y más irrealizable es identificar a qué elementos atiende el observador. Otro ejemplo de esta dificultad se evidencia más claramente al comparar los procedimientos usados para evaluar realce, condicionamiento observacional y emulación de meta. Conceptualmente, en el caso de condicionamiento observacional, lo importante debe ser la asociación entre un manipulandum y la consecuencia; en el caso del realce, debe ser solamente el primero (resaltado por el demostrador) y en el caso de emulación de meta, debería ser solamente la segunda. Sin embargo, empíricamente, el manipulandum casi siempre está relacionado con la consecuencia Por su parte, esta última casi nunca es presentada de manera aislada. Por ejemplo, un trabajo reciente de Klein y Zentall (2003), en el cual se diseñó una situación de emulación de meta, involucró el movimiento de una compuerta y la activación del dispensador de alimento como un control para evaluar verdadera imitación. Evidentemente, este procedimiento permite la relación entre dos estímulos, lo cual se confundiría con condicionamiento observacional; no obstante, el hecho de que la respuesta evaluada implicara la dirección del desplazamiento (izquierdo vs. derecho) y no el simple desplazamiento les permitió hablar de un control de meta. Sin embargo, esta relación entre estímulos puede ser indeseable en otras situaciones, por lo que el control empleado por Zentall y Hogan (1976), en el cual presentan al demostrador solo consumiendo el alimento, parece más adecuado.

De esta manera, no solo es difícil a nivel conceptual separar las diferentes categorías, sino que en los reportes experimentales se propone la evaluación de una categoría y la descripción del procedimiento puede no reflejar fielmente tal evaluación. O bien, la descripción de los procedimientos experimentales es tan ambigua que no permite identificar con exactitud cuál fue el proceso que verdaderamente evaluaron los autores. 
A pesar de todo esto, la existencia de las diferentes maneras de como la conducta de un organismo puede afectar la conducta de otros es incuestionable, y la taxonomía previamente descrita puede ser útil para recordar que, en un procedimiento de aprendizaje social, el observador puede atender a diferentes estímulos, programados por el experimentador o no, y estos deben ser llevados a consideración. Sin embargo, esta taxonomía en sí misma no es suficiente para contestar algunas preguntas importantes sobre aprendizaje social. Por ejemplo, los investigadores que actualmente trabajan en esta área aún no saben con certeza cómo la conducta "es transmitida" de un individuo a otro, cuando el aprendizaje social tiene lugar en contextos naturales o aún bajo condiciones controladas en el laboratorio; así, a la fecha no se tiene claridad sobre los "mecanismos" de aprendizaje involucrados cuando se evalúa un proceso de aprendizaje social o sobre cómo las interacciones sociales contribuyen a la difusión o mantenimiento de conductas que juegan un papel importante en la sobrevivencia y reproducción de los animales que viven en libertad (Galef, 1988).

Como puede advertirse, a pesar de que el aprendizaje social en situaciones apetitivas ha recibido mucha atención, aún existe gran confusión tanto a nivel conceptual como a nivel empírico. Esta confusión parece ser aún más severa para las situaciones de aprendizaje social con control aversivo.

\section{Los problemas inherentes a las situaciones aversivas}

El primer problema es diseñar con control aversivo algunas situaciones que han sido dibujadas en situaciones apetitivas, dado que la identificación de la consecuencia per se es difícil, pues en muchos casos, consiste en la ausencia del estímulo aversivo; en otros casos, es difícil identificar qué controla la respuesta del demostrador: una contingencia de castigo o reforzamiento negativo, por ejemplo. Por ende, la relación respuesta-reforzador resulta ser muy frágil y, por ello, difícil de identificar por parte del observador.
En relación con la ausencia del estímulo aversivo, se tiene que cuando un demostrador es expuesto a contingencias de evitación y responde eficientemente, evitando todas las descargas eléctricas, puede resultar muy difícil para el observador identificar la contingencia respuesta-reforzador, porque la consecuencia frecuentemente no es tangible. Como ya se mencionó, esa contingencia es fundamental para hablar de un proceso imitativo. Por lo tanto, si el observador emite la misma respuesta que el demostrador, resulta difícil clasificar el proceso como imitación, ya que el observador solo ve la respuesta. El proceso implicado tal vez pueda ser clasificado como facilitación social, realce de estímulos o copia.

En relación con la variable que controla la respuesta del demostrador, el problema es ilustrado más claramente en una contingencia de castigo, la cual implica que el sujeto emite una respuesta y recibe una consecuencia que disminuye la probabilidad de esta respuesta; si esta consecuencia va acompañada de un segundo estímulo, es muy probable que el sujeto se aleje de este estímulo. Esta situación implica dos procesos: el castigo de la respuesta que produjo la consecuencia y el reforzamiento de cualquier respuesta que evite la presentación de esta consecuencia y de sus estímulos asociados. Por ende, en situaciones de aprendizaje social en las que esté presente el castigo, es más complicado identificar cuál de las contingencias está identificando el observador. En el estudio de Bunch y Zentall (1980), mencionado anteriormente, el observador ve a un demostrador que se acerca a una vela, se quema y se aleja; en este caso, la respuesta de acercarse a vela es castigada y la respuesta de alejarse, consecuentemente, es reforzada negativamente. Así, cuando el observador no se acerca a la vela, no se sabe cuál contingencia aprendió y, por tanto, se desconoce el proceso de aprendizaje social involucrado.

El segundo problema es inherente a la definición de los diferentes procesos, ya que las categorías o procesos de aprendizaje social parecen haber sido delimitados ad hoc para situaciones apetitivas, las cuales implican respuestas de acercamiento y contacto con los estímulos involucrados. Así, situaciones de control aversivos que involucran respuestas 
de alejamiento de estímulos no son naturalmente contempladas por esa taxonomía, tal es el caso de las contingencias de castigo. Por ejemplo, para que hubiera realce de estímulos con contingencias de castigo, se requeriría que la respuesta del demostrador resalte un estímulo y que ello disminuya la probabilidad de que el observador interactúe con el estímulo. Así, si el observador es expuesto a un demostrador que al presionar una palanca recibe una descarga eléctrica y consecuentemente deja de acercarse a la palanca, el proceso de realce implica que el observador no se acerque a la palanca porque la conducta del demostrador resaltó la zona de la palanca, lo cual per se es inconsistente, pues icómo puede el observador alejarse de un estímulo que su demostrador señaló como relevante? Para que un observador se alejara de un estímulo resaltado sería necesario que hubiera una asociación entre el estímulo y la consecuencia, lo cual descartaría un proceso de realce.

Se concluye que en situaciones aversivas el observador es expuesto a una relación respuestareforzador, pero que la naturaleza del estímulo reforzante es diferente en cada una de las situaciones. Mientras en la apetitiva, se presenta un estímulo que el observador ve, en la aversiva se elimina un estímulo que generalmente no es visible para el observador, de tal manera que, para ser adaptativo, el organismo debe aprender a evitar estímulos aversivos al ser expuesto a las respuestas provocadas por los estímulos en otros y no a los estímulos en sí mismos.

\section{La imitación: sus elementos esenciales}

Por su parte la imitación, que es uno de los procesos de aprendizaje social más complejos y de mayor interés para los psicólogos experimentales, requiere que el observador tome el lugar del demostrador para identificar su perspectiva, como en los experimentos en los que la direccionalidad de la respuesta es el elemento a reproducirse; al respecto Heyes y sus colaboradores han realizado experimentos en los que el demostrador desplaza un joystick a la derecha o la izquierda en t1, en t 2 el observador debe desplazar el operando en la misma dirección en que lo hizo su demostrador para recibir reforzador (Heyes \& Dawson, 1990; Heyes, Dawson \& Nokes, 1992). Recientemente, Mui, Hazelgrove, Pearce y Heyes (2008) requieren una respuesta de igualación casi inmediata por parte de los observadores a la respuesta del demostrador. De igual manera, el observador debe identificar cuál es la respuesta efectiva del demostrador para producir el reforzador y debe ser capaz de traducir esta información visual en los movimientos que le permitan realizar la respuesta de igualación.

Como ejemplo de discriminación de la propia conducta, Beninger, Kendall y Vanderwolff (1974) enseñaron a ratas a presionar cuatro palancas para recibir alimento. Cuatro respuestas distintas fungieron como estímulos discriminativos: acicalarse, caminar, pararse en dos patas o estar inmóvil. La palanca correcta en cada ensayo cambiaba dependiendo de cuál de estas respuestas la rata estuviera emitiendo en el momento en que sonara un tono. La respuesta de presionar la palanca estuvo bajo el control de las respuestas que estaban emitiendo en el momento en que sonó el tono, lo cual fue evidenciado por índices de discriminación por arriba del nivel de oportunidad. Esos resultados sugieren que los sujetos son capaces de discriminar sus propios movimientos, pero no aclara cómo se da la traducción de lo que ven a lo que hacen.

Una aproximación que intenta dar cuenta de la manera como ocurre esta traducción es la de las neuronas espejo. La propuesta es que hay un mecanismo que permite una igualación directa entre la entrada visual de una acción y su posterior ejecución; cuando un organismo ve que otro realiza una acción y obtiene una consecuencia, se activan neuronas que permiten al segundo organismo tener una representación motora de esa acción y una anticipación sensorial de las consecuencias que obtendrá con la reproducción de la acción observada; así, la mera observación de un acto motor determina la activación de su representación motora, permitiendo que el acto se manifieste en el observador. Estos actos motores pueden existir ya en el repertorio del observador y se modifican (actualizan) en función de la acción del demostrador y de las consecuencias que produce (Rizzolatti, Fogassi \& Gallese, 2001). 
En el caso de situaciones aversivas, el mecanismo inicia cuando un observador identifica en otro organismo señales de disgusto tales como las faciales, lo cual produce la activación de neuronas que le permiten tener una representación de disgusto y otra de la acción motora, a partir de lo que el otro organismo hace (Gallese, Keysers \& Rizzolatti, 2004).

Para concluir, se considera que en aprendizaje social aún hay muchas respuestas por resolver tanto de carácter conceptual como empírico, que es necesario el diseño de experimentos cuya metodología permita aislar confiablemente los elementos involucrados para tratar de identificar su papel en el proceso a evaluar y que se requiere de una taxonomía que permita incorporar los procesos de aprendizaje social en contextos aversivos (Arriaga et al., 2006; Shettleworth, 2010; Zentall, 2006).

\section{Referencias}

Arriaga, J. C. P., Ortega-Saavedra, G., Meza, G., Huichán, F., Juárez, E., Rodríguez, A., et al. (2006). Análisis conceptual del aprendizaje observacional y la imitación. Revista Latinoamericana de Psicología, 38, 87-102.

Avital, E. \& Jablonka, E. (1994). Social learning and the evolution of behaviour. Animal Behaviour, 48, 1195-1199.

Beninger, R. J., Kendall, S. B. \& Vanderwolf, C. H. (1974). The hability of rats to discriminate their own behaviors. Canadian Journal of Psychology, 28, 79-91.

Bond, N. W. (1984). The poisoned partner effect in rats: Some parametric considerations. Animal Learning and Behavior, 29, 543-550.

Bunch, G. B. \& Zentall, T. R. (1980). Imitation of a passive avoidance response in the rat. Bulletin of the Psychonomic Society, 15, 73-75.

Catania, A. C. (1989). Learning. Engleewood Cliffs, NJ: Prentice-Hall.

Coombs, S. S., Revusky, S. \& Lett, B. T. (1980). Long delay taste aversion learning in an unpoisoned rat: Exposure to a poisoned partner as the US. Learning and Motivation, 11, 256-266.
Cook, M. \& Mineka, S. (1990). Selective associations in the observational conditioning of fear in rhesus monkeys. Journal of the Experimental Psychology: Animal Behavior Processes, 16, 372-389.

Dickinson, A. (1980). Contemporary animal learning theory. Cambridge: Cambridge University Press.

Domjam, M. (2009). The principles of learning and behavior (6. ${ }^{\mathrm{a}}$ ed.). New York: Cengage Learning Inc.

Galef, B. G., Jr. (1988). Communication of information concerning distant diets in a social, central-place foraging species: Rattus norvegicus. En T. R. Zentall \& B. G. Galef, Jr. (Eds.), Social learning: Psychological and biological perspectives (pp. 119-140). Hillsdale, NJ: Lawrence Eribaum Associates Publishers.

Gallese, V., Keysers, C. \& Rizzolatti, G. (2004). A unifying view of the basis of social cognition. Trends in Cognitive Sciences, 8, 396-403.

Herrnstein, J. R. (1970). On the law of effect. Journal of the Experimental Analysis of Behavior, 13, 243-266.

Heyes, C. M. (1994). Social learning in animals: Categories and mechanisms. Biological Reviews, 69, 207-231.

Heyes, C. M. (1996a). Introduction: Identifying and defining imitation. En C. M. Heyes \& B. G. Galef, Jr. (Eds.), Social learning in animals: The roots of culture (pp. 211- 220). San Diego, CA: Academic Press.

Heyes, C. M. (1996b). Genuine imitation? En C. M. Heyes \& B. G., Galef, Jr. (Eds.), Social learning in animals: The roots of culture (pp. 371-389). San Diego, CA: Academic Press.

Heyes, C. M. \& Dawson, G. R. (1990). A demonstration of observational learning in rats using a bidirectional control. Quarterly Journal of Experimental Psychology, 42B, 59-71.

Heyes, C. M., Dawson, G. R. \& Nokes, T. (1992). Imitation in rats: Initial responding and transfer evidence. Quarterly Journal of Experimental Psychology, 45B, 229-240.

Honig, W. K. (1966). Operant behavior: Areas of research and applications. New York: Meridith Corporation.

Honig, W. K. \& Staddon, J. E. R. (1977). Handbook of operant behavior. Englewood Cliffs, NJ: PrenticeHall.

Iversen, I. H. \& Lattal, K. A. (1991) Experimental analysis of behavior (Vols. 1 y 2). Amsterdam: Elsevier. 
Klein, D. E. \& Zentall, T. R. (2003). Imitation and affordance learning by pigeons (Columba livia). Journal of Comparative Psychology, 117, 414-419.

Lavin, M. J., Freise, B. \& Coombs, S. (1980). Transferred flavor aversions in adult rats. Behavioral and Neural Biology, 28, 15-33.

Mackintosh, N. J. (1983). Conditioning and associative learning. New York: Oxford University Press.

Mowrer, O. H. (1947). On the dual nature of learning: A reinterpretation of "conditioning" and "problemsolving". Harvard Educational Review, 17, 102-150.

Mowrer, O. H. \& Lamoreaux, R. R. (1942). Avoidance conditioning and signal duration: A study of secondary motivation and reward. Washington: Evanston III., The American Psychological Association, Psychological Monographs, 54 (5, Whole no. 247).

Mui, R., Hazelgrove, M., Pearce, J. \& Heyes, C. (2008). Automatic imitation in budgerigars. Proceedings of the Royal Society of London B, 275, 2547-2553.

Nicol, C. J. \& Pope, S. J. (1992). Effects of social learning on the acquisition of discriminatory keypecking in hens. Bulletin of Psychonomic Society, 30, 293-296.

Papworth, S., Bosë, A. -S., Barker, J., Schel, A. M. \& Zuberbühler, K. (2008). Male blue monkeys alarm call in response to danger experienced by other. Biology Letters, 4, 472-475.

Rescorla, R. A. (1988). Behavioral studies of Pavlovian conditioning. Annual Review of Neuroscience, 11, 329-352.

Rescorla, R. A. \& Wagner, A. R. (1972). A theory of Pavlovian conditioning: Variations in the effectiveness of reinforcement and nonreinforcement. En A. H. Black \& W. F. Prokasi (Eds.), Classical conditioning II: Current research and theory (pp. 6499). New York: Appleton-Century-Crofts.
Riess, D. (1972). Vicarious conditioned acceleration: Successful observational learning of an aversive pavlovian stimulus contingency. Journal of the Experimental Analysis of Behavior, 18, 181-186.

Rizzolatti, G., Fogassi, L. \& Gallese, V. (2001). Neurophysiological mechanisms underlying the understanding and imitation of action. Nature Reviews Neuroscience, 2, 661-670.

Sanavio, E. \& Savardi, U. (1979). Observational learning of a discriminative shuttlebox avoidance by rats. Psychological Reports, 44, 1151-1154.

Shettleworth, S. J. (2010). Cognition, evolution, and behavior. New York: Oxford University Press.

Thorndike, E. L. (1898). Animal intelligence: An experimental study of the associative processes in animals. Psychological Review Monograph Supplements, 2 (4, Whole no. 8). New York: Macmillan.

Whiten, A. \& Ham, R. (1992). On the nature an evolution of imitation in the animal kingdom: Reappraisal of a century of research. Advances in the Study of Behavior, 21, 239-283.

Zajonc, R. B. (1965). Social facilitation. Science, 149, 269-274.

Zentall, T. R. (1996). An analysis of imitative learning in animals. En C. M. Heyes \& B. G., Galef, Jr. (Eds.), Social learning in animals: The roots of culture (pp. 221-243). San Diego, CA: Academic Press.

Zentall, T. R. \& Hogan, D. E. (1976). Imitation and social facilitation in the pigeon. Animal Learning \& Behavior, 4(4), 427-430.

Zentall, T. R. \& Levine, J. M. (1972). Observational learning and social facilitation in the rat. Science, $178,1220-1221$ 